\title{
Problemas asociados al tabaquismo en Chile
}

\author{
HUGO AMIGO(1) y MARCIA ERAZO(2)
}

\section{Prevalencia de tabaquismo}

E1 tabaquismo es la principal causa de enfermedad, discapacidad y muerte prematura prevenible'. A nivel mundial, se le han atribuido más casos de muerte por cáncer que a cualquier otro cancerígeno conocido, y que además de cáncer también produce muertes por enfermedades cardiovasculares y respiratorias 2,3 .

El consumo de tabaco es un hábito ampliamente difundido en el mundo entero, estimándose que más de mil millones de personas son fumadoras y que cada año, alrededor de treinta millones de adultos jóvenes adquieren el hábito ${ }^{4}$. En América Latina, el hábito tabáquico es muy frecuente, calificándose que la mayoría de los países se encontraban en estadio 2, es decir, con un claro aumento en la prevalencia de tabaquismo en los hombres, un leve incremento en las mujeres y una mortalidad atribuible al tabaco que aún no reflejaba el aumento en la prevalencia de su consumo. Ninguno de los países analizados había avanzado a la fase 3 , caracterizada por una disminución de la prevalencia de tabaquismo en hombres y gran aumento en mujeres. En el otro extremo, únicamente Paraguay se encontraba emergiendo de fase uno, caracterizada por presentar baja prevalencia en hombres ${ }^{5}$.

Varias publicaciones señalan que Chile, a mediados de los noventa, era un país con alta prevalencia de tabaquismo ${ }^{6-8}$. Estudios realizados posteriormente indican que ésta se ha mantenido estable, por encima de $40 \%$ en la población general, observándose además un incremento de este hábito en las mujeres y una disminución en la edad de inicio de su consumo ${ }^{9}$. Sin embargo, aunque esta prevalencia sea elevada, el consumo promedio de tabaco entre los fumadores es moderado, en comparación con otros países que exhiben un promedio de consumo considerablemente mayor y sus niveles de prevalencia, incluso son menores ${ }^{10.11}$.

En la Figura 1, se presenta la prevalencia de tabaquismo de acuerdo a los diversos estudios realizados en el país. La Encuesta Nacional de Salud consignó que $42 \%$ de los chilenos son fumadores actuales, $33 \%$ fumadores ocasionales y $9 \%$ fumadores de un cigarrillo diario. Se constató además que aún se observan diferencias significativas entre hombres y mujeres ( $48 \%$ y $37 \%$, respectivamente). La prevalencia de fumadores actuales muestra estrecha relación con la edad, siendo mayor a edades menores $(55 \%$ en menores de 24 años y $11 \%$ en mayores de 65 ). Consistentemente, los hombres son más fumadores en todas las edades, con máxima prevalencia entre 20 y 30 años $(65 \%)$ que luego desciende en forma sostenida hasta llegar al menor nivel, a los 80 años (menos del $10 \%$ ). En las mujeres la prevalencia de tabaquismo actual presentó una distribución bimodal, con un primer peak entre los 20 y 25 años (59\%) y otro entre los 40 y 50 años (40\%), manteniendo la edad fértil con valores inferiores a $30 \%$. En promedio los chilenos fuman 8,2 cigarrillos diarios, superando los hombres en 1,5 cigarrillos/día a las mujeres ${ }^{12}$.

Otro antecedente lo provee el estudio de la cohorte de nacidos vivos en la ciudad de Limache entre 1975 y 1978 , en el que se entrevistó a más de 1230 adultos jóvenes. Se observó que casi $70 \%$ de los hombres y $50 \%$

\footnotetext{
(1) Facultad de Medicina. Universidad de Chile.

(2) Programa de Doctorado en Salud Pública. Universidad de Chile.
} 
Figura 1. Prevalencia de tabaquismo en Chile de acuerdo a los principales estudios efectuados en el país

\begin{tabular}{|c|c|c|c|}
\hline & Título & Metodología & Resultados \\
\hline $\begin{array}{l}\text { Medina E, Pascual JP, } \\
\text { Cumsille F et als } \\
\text { Rev Med Chile 1986; } \\
114: 257-62 \text {. }\end{array}$ & $\begin{array}{l}\text { Encuesta de tabaquismo en } \\
\text { la población general de } \\
\text { Santiago }\end{array}$ & $\begin{array}{l}\text { Encuesta telefónica } \\
\text { a } 1.000 \text { habitantes } \\
\text { de Santiago, utilizando } \\
\text { el formulario de la } \\
\text { encuesta básica de } \\
\text { consumo de la } \\
\text { American Cancer Society }\end{array}$ & $\begin{array}{l}\text { - El } 41,2 \% \text { de los } \\
\text { habitantes son } \\
\text { fumadores actuales, } \\
44 \% \text { en hombres y } \\
39 \% \text { en mujeres, de ellos } \\
\text { tres cuartos lo consumen } \\
\text { en forma diaria. } \\
\text { - Las más altas } \\
\text { prevalencias se } \\
\text { observan en los } \\
\text { más jóvenes. } \\
\text { - El consumo promedio } \\
\text { de cigarrillos } \\
\text { es de } 10 \text { diarios. } \\
\text { - No se observaron } \\
\text { diferencias } \\
\text { socioeconómicas } \\
\text { en el consumo. }\end{array}$ \\
\hline
\end{tabular}

Medina E, Kaempfer A, Tabaquismo en Santiago,

Cornejo E,

Hernández E.

Rev Méd Chile 1995;

123: 652-8

\section{3-94}

(n)
Encuesta a 4.700

personas que

vivian en 12

comunas de Santiago,

sobre frecuencia

de eventos de salud

y características de

la atención de

salud recibida

- El 37,3\% de la población de 15 años y más es fumador actual.

- El 26,9\% lo hace en forma diaria y el $10,4 \%$ son fumadores ocasionales.

- El 15,5\% son ex fumadores

- Se observó que en el nivel socioeconómico bajo existe la más alta prevalencia ( $52 \%$ ).

$-40,6 \%$ fumadores regulares.

Se encuestaron

3120 sujetos, las enfermedades no 25-45 años de edad, Valparaíso, acerca del ransmisibles: Metodologia y resultados globales de hábito tabáquico, actividad fisica y otros hábitos.

Encuesta a 1959 personas en Viña del Mar y Valparaíso, usando un cuestionario sobre consumo de tabaco, basado en la encuesta de tabaquismo de la OMS
- La prevalencia de tabaquismo en mayores de 10 años fue $36,5 \%$ y en los mayores de 15 años, $40,8 \%$.

- La prevalencia en hombres es 49,4\% y $35 \%$ en mujeres.
Ministerio de Salud
Resultados Primera Encuesta Nacional de Salud 2004
$48 \%$ de los hombres y $37 \%$ de las mujeres habian fumado el último mes, el promedio de cigarros fumados es 8,2 la intensidad. 


\section{Continuación Figura 1}

\begin{tabular}{|c|c|c|c|}
\hline $\begin{array}{l}\text { Gobierno de Chile } \\
\text { Conace }\end{array}$ & $\begin{array}{l}\text { El consumo de cigarrillos } \\
\text { en Chile (Estudios } \\
\text { Nacionales de Drogas en } \\
\text { Población General de Chile } \\
\text { 1999-2004) o }\end{array}$ & $\begin{array}{l}\text { Entrevistas cara a cara } \\
\text { en hogares, en } \\
\text { población de } 12 \text { a } 64 \\
\text { años de ambos sexos, } \\
\text { muestra nacional en } \\
\text { todas las regiones del pais. }\end{array}$ & $\begin{array}{l}\text { Entre } 1994 \text { a } 2004 \text { los } \\
\text { hombres mantienen } \\
\text { niveles estables de } \\
\text { consumo. En } 2004 \text { era } \\
\text { de } 44,83 \% \text { sobre el } 43 \% \\
\text { y en las mujeres se } \\
\text { observa un aumento } \\
\text { alcanzando en } 2004, \text { a } \\
40,37 \% \text { se indica que } \\
\text { el promedio de cigarrillos } \\
\text { fumados por dia era de seis. }\end{array}$ \\
\hline $\begin{array}{l}\text { Amigo H, Oyarzún M, } \\
\text { Bustos } \mathrm{P} \text {, Rona R, } \\
\text { Cohorte de Limache } \\
\text { 2003-2004 en procero } \\
\text { de publicación. }\end{array}$ & $\begin{array}{l}\text { Respiratory consequences } \\
\text { of light and moderate } \\
\text { smoking in young adults. }\end{array}$ & $\begin{array}{l}\text { Se entrevistaron } \\
1.232 \text { jovenes nacidos } \\
\text { en la maternidad de } \\
\text { Limache entre } 1975 \text { a } \\
1978 \text {, seguiendo un } \\
\text { cuestionario estructurado } \\
\text { basado en el Estudio } \\
\text { Europeo de enfermedades } \\
\text { respiratorias. }\end{array}$ & $\begin{array}{l}\text { Un } 67,7 \% \text { de los hombres } \\
\text { y un } 49,4 \% \text { de las mujeres } \\
\text { habian fumado el mes } \\
\text { anterior a la entrevista. }\end{array}$ \\
\hline
\end{tabular}

de las mujeres son actualmente fumadores. Este estudio confirma que la intensidad del consumo es moderada, aunque, por otro lado, revela una inversión del diferencial por sexo, con una mediana de consumo de 3 cigarrillos/ día en hombres y 4 en mujeres ${ }^{13}$.

Las encuestas realizadas por la Comisión Nacional de Control de Estupefacientes (Conace) permiten analizar la tendencia del tabaquismo en la población general de 12 a 64 años. En el último decenio, la prevalencia en los hombres ha permanecido estable (alrededor de un $45 \%$ ), mientras que en las mujeres se ha incrementado en un $6 \%$, llegando en el año 2004 a una tasa de prevalencia de $40 \%$. En los adolescentes, se observó que ha permanecido estable en ambos sexos ${ }^{14}$.

\section{Efectos del tabaquismo sobre la salud}

Los efectos del tabaquismo sobre la salud son variados. La mayoría de los estudios se concentra en los efectos a largo plazo por enfermedades como cáncer pulmonar, enfermedad obstructiva pulmonar, infarto del miocardio y accidente vascular encefálico, entre otras $^{15-17}$. Se ha establecido que un tercio de todas las muertes por cáncer y la mitad de los infartos en adultos de media edad en países occidentales, pueden atribuirse al consumo de cigarrillos. La morbilidad atribuible al tabaco viene aumentando progresivamente, calculándose una fracción atribuible de $85 \%$ para cáncer y enfermedad pulmonar obstructiva crónica y de un $35 \%$ para enfermedades cardiovasculares en los Estados Unidos de Norteamérica ${ }^{18}$.

Pese a la evidencia sobre la importancia del efecto del tabaquismo, los estudios causales en el país han sido limitados siendo que los últimos trabajos publicados se remontan a la época de los ochenta ${ }^{19}$. En este contexto, recientemente estamos conduciendo un estudio multicéntrico sobre fracción atribuible al tabaco en tres patologías, el cual confirma los valores atribuibles estimados a nivel mundial ${ }^{20}$.

Varios estudios internacionales también confirman la asociación entre el hábito de fumar y sintomas respiratorios como sibilancia, tos crónica y disnea y con la disminución de la función pulmonar ${ }^{21-23}$. No obstante, hay escasos estudios referentes al papel del tabaquismo moderado, probablemente porque la atención ha estado concentrada en las enfermedades que necesitan una preocupación urgente por su gravedad. En el estudio de Limache antes citado pudimos verificar una marcada asociación entre fumar y sintomas respiratorios aún en los que fumaban menos de 5 cigarros por día ${ }^{13}$. 


\section{Características del tabaco consumido en el país}

En Chile, más del $95 \%$ del tabaco consumido es industrialmente manufacturado, existiendo dos marcas más comerciales que concentran las ventas en el mercado. El contenido de alquitrán en estas marcas es de 14,64 y $8,9 \mathrm{mg} / \mathrm{cig}$. $Y$ de nicotina es 1,36 y $0,70 \mathrm{mg} / \mathrm{cig}$. Desde 1994, el $100 \%$ de los cigarrillos comercializados poseen filtro, el precio promedio de una cajetilla de 20 unidades con impuesto incluido es de USD 1.19 equivalente en la marca nacional y de USD 1.40 en el importado. Ambos valores son inferiores al precio de venta en países desarrollados 24 .

\section{Costos en salud asociados al tabaquismo}

Se ha estimado que, en países desarrollados, los gastos en enfermedades derivadas del tabaquismo corresponderían al 15\% del presupuesto total de salud. En el estudio multicéntrico previamente citado, hemos estimado los costos unitarios derivados del diagnóstico y primer año de tratamiento de la enfermedad pulmonar obstructiva crónica, cáncer pulmonar e infarto al miocardio.

La medición de los costos para estas tres patologías requirió -en una primera etapaidentificar las prestaciones asociadas al diagnóstico y tratamiento en cada una de las fases de la enfermedad. Para tal efecto, se confeccionaron guías diagnóstico-terapéuticas detalladas para cada patología, las que posteriormente se valorizaron utilizando como referencia los aranceles del Fondo Nacional de Salud (Fonasa). Las prestaciones consideradas en cada patología incluyen los procedimientos diagnósticos y tratamientos aplicados durante el primer año, para un paciente representativo y con un nivel de atención estándar. El estudio representa un esfuerzo orientado a determinar el "piso" de los recursos financieros involucrados en el diagnóstico y tratamiento de las principales patologías asociadas al consumo de tabaco.

En la Figura 2, se observan los costos unitarios estimados para un caso incidente durante el primer año de tratamiento, según grado de severidad al momento del diagnóstico, para EPOC, Cáncer de pulmón e Infarto Q y No Q.

Figura 2. Costos unitarios (USD) de Epoc, cáncer pulmonar e infarto al miocardio

\begin{tabular}{lrrr}
\hline Patologías & Leve & Moderado & Grave \\
\hline Cáncer * & $4.638,3$ & 13.797 & 21.016 \\
Epoc & 214,6 & 388,8 & $4.157,8$ \\
Infarto con ST & & $6.240,5$ & \\
elevación & & & \\
de segmento & & $7.537,0$ & \\
lnfarto sin & & & \\
elevación del & & & \\
segmento & & & \\
\hline
\end{tabular}

* En el caso del cáncer la subdivisión de casos leves corresponde a los estadios 1 y 2 , moderados $3 \mathrm{~A}$ y $3 \mathrm{~B}$ y severo estadios 4 con y sin compromiso del sistema nervioso central.

\section{REFERENCIAS}

1.- EZZATI M, LÓPEZ AD, RODGERS A, VANDER HOORN S, MURRAY C J. Comparative risk assessment collaborating group. Selected major risk factors and global and regional burden of disease. Lancet $2002 ; 360$ (9343): $1347-60$.

2.- PETO R, LÓPEZ A D, BOREHAM J, THUN M, HEATH C J R. Mortality from smoking in developed countries 1950-2000: Indirect estimates from National Vital Statistics, Oxford, Oxford University Press, 1994.

3.- PARKIN D M, PISANI P, MASUYER E. Tobaccoattribuitable cancer burden: A global review. En: Lu R, Mackay J, Niu S, Peto R, eds. Tobacco: The growing Epidemic, London, Springer-Verlag, 2000, pp. $81-4$.

4.- WHO. The World Health Report 1995. Bridging the gap. Ginebra.WHO: 1995.

5.- DA COSTA E SILVA V L, KOIFMAN S. Smoking in Latin America: a major public health problem. Cad Saude Publica 1998; 14 Suppl 3: 99-108.

6.- MEDINA E, KAEMPFER A, CORNEJO E, HERNÁNDEZ E. 'Tabaquismo en Santiago, 199394. Rev Méd Chile 1995; 123:652-8.

7.- GUTIERREZ M, RIOSECO F, ROJAS A et al. Prevalencia de tabaquismo en la población general de Valparaiso y Viña del Mar. Rev Méd Chile 1995; 123: 250-6.

8. JADUE L, VERGARA J, ESCOBAR $M$, DELGADO I, GARRIDO C, LASTRA P, ESPEJO F, PERUGA A. Factores de riesgo para las enfermedades no trasmisibles: Metodología y 
resultados globales del programa CARMEN. Rev Méd Chile 1999; 127: 1004-13.

9.- ALVARADO R, TALAVERA G. La epidemia de tabaquismo en Chile: Evolución durante la última década. Rev Chil Salud Pública 2003; 7 (2):69-73,

10.- WHO-TOBACCO FREE INICIATIVE, 2002, disponible en: http://fi.wpro.who.int/ eco consumption.asp?nation code $=231$ (visitado, 20 de abril de 2005).

11.- WHO 2003 disponible en: http:// www.nationmaster.com/graph-T/hea_tob_cig_con (consultado el 20 de abril de 2005).

12.- ENCUESTA NACIONAL DE SALUD 2003. Disponible en: http:/Www.minsal.cl (consultado 20 abril de 2005).

13. AMIGO H, OYARZÚN M, BUSTOS P, RONA R J. Respiratory consequences of light and moderate smoking in young adult in Chile, En proceso de publicación.

14.- GOBIERNO DE CHILE. CONACE. El consumo de cigarrillos en Chile. Estudio nacional de drogas en población general de Chile, 1994-2004, Santiago Chile.

15.- SASCO A J, SECRETAN M B, STRAIF K. Tobacco smoking and cancer: a brief review of recent epidemiological evidence. Lung Cancer 2004; 45(Suppl 2): S3-S9.

16.- MAHONEN M S, MCELDUFF P, DOBSON A J, KUULASMAA K A, EVANS A E; WHO MONICA Project. Current smoking and the risk of non-fatal myocardial infarction in the WHO MONICA Project populations. Tob Control 2004; 13(3): 244-50.

17.- JAEN DIAZ J 1, DE CASTRO MESA C, GONTAN
GARCÍA SALAMANCA M J, LÓPEZ DE CASTRO $F$. Prevalence of chronic obstructive pulmonary disease and risk factors in smokers and ex smokers. Arch Bronconeumol 2003; 39(12): 554-8.

18.- ANNE M J, MD, MPH; LAWENCE C AN, MD. Tobacco smoking in patients with cardiovascular disease. Current treatment options in cardiovascular medicine 2001, (3), 313-322.

19.- MARTÍNEZ O L, PAREDES R, CARIS L. Epidemiology of bronchopulmonary cancer in Chile. Rev Méd Chile 1985; 113(1): 48-54.

20.- INFORME DE AVANCE. Estudio multicéntrico costos de atención de atención médica en tres patologias atribuible al consumo de tabaco, en cuatro países de América Latina, Santiago, Chile 2004.

21.- LINDSTROM M, KOTANIEMI J, JONSSON E, LUNDBACK B. Smoking, respiratory symptoms, and diseases : a comparative study between northern Sweden and northern Finland: report from the FinEsS study. Chest 2001; 119(3): 852-61.

22.- LANGHAMMER A, JOHNSEN R, HOLMEN J, GULSVIK A, BJERMER L. Cigarette smoking gives more respiratory symptoms among women than among men. The Nord-Trondelag Health Study (HUNT). J Epidemiol Community Health 2000; 54 : $917-22$.

23.- LANGHAMMER A, JOHNSEN R, GULSVIK A, HOLMEN TL, BJERMER 1. Sex differences in lung vulnerability to tobacco smoking. Eur Respir $J$ 2003; $21: 1017-23$.

24. - WHO GLOBAL STATUS REPOR'T-CHLE-1997. http:/www.edc.gov./tobacco/who/chile.htm (consultado el 21 de abril de 2005 ).

Usted puede comentar éste y otros articulos publicados en la Revista Chilena de Salud Pública, enviando un correo electrónico a revistasp@med.uchile.cl 